\title{
SOFRIMENTO DOS PROFISSIONAIS QUE ATUAM NO SETOR DE ONCOLOGIA EM UM HOSPITAL PÚBLICO DE JOINVILLE, SC
}

\author{
SUFFERING OF PROFESSIONALS THAT ACT IN THE ONCOLOGY SECTOR AT A PUBLIC HOSPITAL \\ IN JOINVILLE, SC, BRAZIL
}

\author{
Arlene Laurenti Monterrosa Ayala ${ }^{a}$, Amábile Cristina Rosa Feliciob ${ }^{b}$ Jessica Pachãoc \\ aalayala@bol.com.br, bmabyrosa_@hotmail.com, cjessyca_pachao@hotmail.com \\ Associação Educacional Luterana Bom Jesus/lelusc - Joinville (SC), Brasil
}

Data de recebimento do artigo: 24/11/2016

Data de aceite do artigo: 15/02/2017

\section{RESUMO}

Introdução: É possível constatar que o trabalho em saúde, especialmente em oncologia, exige do trabalhador o trato cotidiano com o sofrimento alheio e a morte. Essa exigência traz diversas implicaçóes para a saúde física e mental deles, para além de outras atividades de serviço. Objetivo: O estudo teve como propósito identificar o sofrimento e a presença de Transtornos Mentais Comuns (TMC) em quinze profissionais que atuam em uma unidade que atende pacientes oncológicos de um hospital geral no município de Joinville/SC. Materiais e métodos: Trata-se de estudo descritivo que utilizou como técnica de coleta de dados um questionário com perguntas abertas e fechadas, especialmente desenvolvido para conhecer as características dos profissionais em estudo e para a avaliaçáo da presença de Transtornos Mentais Comuns (TMC). Foram aplicadas vinte perguntas extraídas do Self-reporting questionnaire (SRQ-20). Resultados: Os entrevistados tinham média de idade de 41,46 anos, sendo a maioria mulheres com menos de cinco anos trabalhados na unidade oncológica. Foram encontrados fatores que influenciam o trabalho dos profissionais no setor de oncologia, que podem ser descritos como aqueles relacionados às condiçóes de trabalho, dificuldade em lidar com a terminalidade do paciente oncológico e relação com familiares. A partir da aplicaçáo do questionário SRQ-20, identificou-se que 20\% dos trabalhadores apresentaram rastreamento positivo para TMC e 73,34\% dos entrevistados afirmaram ter alguma queixa. Conclusão: Tais resultados indicam a necessidade de intervenção, visando melhorar as condiçóes gerais de trabalho e fornecer suporte emocional ao coletivo de trabalhadores.

Palavras-chave: Enfermagem oncológica; transtornos mentais; condiçóes de trabalho.

\section{ABSTRACT}

Introduction: It is possible to verify that the work in health area, especially in oncology, demands from the worker the daily deal with others' suffering and death. This requirement has several implications for them physical and mental health, in addition to other service activities. Objective: This study aims to identify the suffering and presence of Common Mental Disorders (CMD) of fifteen professionals that work in a cancer patient-centered unit from a general hospital in the city of Joinville, state of Santa Catarina. Materials and methods: Descriptive study using as data collection technique a questionnaire with open and closed questions specially carried out to know the characteristics of professionals under study and for the evaluation of presence of Common Mental Disorders (TMC). There were applied twenty questions extracted from the Self-reporting questionnaire (SRQ-20). Results: Interviewees had a mean age of 41,46 years; the majority were women with less than five years working in the oncology unit. Factors that influence the work of professionals in oncology sector were found, which can be described as those related to working conditions, difficulty in dealing with the terminality of cancer patients and relationship with relatives. From SRQ-20 questionnaire, it was identified that $20 \%$ of workers had a positive CT scan and $73.34 \%$ of interviewees reported having some complaint. Conclusion: These results show the need of intervention, aiming to improve the general conditions of work and provide emotional support to these workers.

Keywords: Oncology nursing; mental disorders; work conditions. 


\section{Introdução}

Este estudo teve como propósito identificar, por meio de uma pesquisa descritiva, o sofrimento dos profissionais que atuam na unidade que atende pacientes portadores de câncer de um Hospital Geral do município de Joinville/SC.

De acordo com o Instituto Nacional de Câncer, citando a Organização Mundial de Saúde (OMS) o número global de mortes por câncer subiu de 7,6 milhóes de casos em 2008 para 8,2 milhóes em 2012, principalmente nos países desenvolvidos e em desenvolvimento. Esse aumento se deve em parte pelo aumento da expectativa de vida da população, estilo de vida que predispóe à doença e o desenvolvimento técnico-científico na área oncológica, que ampliou as possibilidades diagnósticas ${ }^{1}$.

Apesar dos avanços no diagnóstico e no tratamento, a mortalidade em decorrência da doença é alta. De acordo com Silva ${ }^{2}$, o câncer traça um longo curso e seu tratamento pode interferir de forma dramática na qualidade de vida das pessoas acometidas por essa enfermidade. Para o autor, cada vez mais os profissionais se veem envolvidos com essa doença, assumindo importante papel na rede de relaçóes interpessoais que ela desencadeia.

É constatado por estudos que cuidar do paciente oncológico é um grande desafio para os profissionais, devido a suas caraterísticas, como: tratamentos prolongados e agressivos com efeitos colaterais, cirurgias mutiladoras, sentimentos de medo, desespero e pânico dos doentes e morte têm sido evidenciados como fatores de estresse e estão associados ao sofrimento psíquico do profissional de enfermagem em oncologia ${ }^{3,4}$.

Oliveira $^{5}$ considera que os profissionais que lidam com pacientes graves, ao perceberem que estáo lidando com seres humanos como eles, parecem experimentar uma vivência de extrema angústia. É difícil se defrontar com pacientes adultos utilizando fraldas, imobilizados, conectados a aparelhos, chorando, às vezes inconscientes, além de outras situaçóes constrangedoras. $\mathrm{O}$ profissional se vê diante da precariedade da existência humana.

Porto et al. ${ }^{6}$, ao identificarem as relaçóes presentes entre os profissionais de saúde que trabalham com pacientes oncológicos no programa de internação domiciliar interdisciplinar, revelam que no cotidiano desses profissionais também se apresentam escutas entre eles para a atenuação de conflitos de opinióes e trocas de respeito e afeto mútuos, auxiliando-os no controle do sofrimento e na satisfação pela atuação em conjunto.

$\mathrm{Franco}^{7}$ refere que os profissionais, especialmente aqueles da área hospitalar, reconhecem a existência de pacientes especiais com os quais se estabelece uma relação estreita, e que a morte desses pacientes pode provocar o luto, caracterizado por sintomas psicológicos e somáticos que causam sofrimento e dor. Esses sintomas compreendem manifestaçóes afetivas, tais como culpa, ansiedade, depressão; manifestaçóes comportamentais como fadiga, choro, baixa autoestima e desamparo; lentidáo do pensamento e da concentração; perda de apetite; distúrbio do sono; queixas somáticas, como dores, náuseas, nó na garganta, palpitaçôes, e sensação de estômago vazio; mudanças na ingestão e suscetibilidade a doenças.

Ainda sobre os sentimentos dos profissionais de saúde frente à situação da terminalidade e do câncer, os resultados do estudo de Faria e Maia ${ }^{8}$ revelaram que $65 \%$ dos profissionais afirmaram sentirem-se diferentes ao atender o paciente nessa situação. Desses, $25 \%$ afirmaram ter sentimentos de sofrimento e tristeza; $17 \%$ referiram sentir-se angustiados; $7 \%$ tiveram sentimentos de impotência; $5,1 \%$, medo; $5,1 \%$, atenção; $5,1 \%$, apreensão; $5,1 \%$, amor $5,1 \%$, vida; $5,1 \%$, humanidade; $5,1 \%$, perda; $5,1 \%$, vulnerabilidade; $5,1 \%$, carência; $5,1 \%$, preocupação.

É possível constatar que o trabalho em saúde, especialmente em oncologia, exige algo do trabalhador: o trato cotidiano com o sofrimento alheio e a morte. Essa exigência traz diversas implicaçóes para sua saúde física e mental, para além de outras atividades de serviço. $\mathrm{O}$ desgaste desse trabalhador decorrente das situaçóes de trabalho, aliado ao desgaste psíquico, é motivo suficiente para o desenvolvimento deste estudo.

\section{Metodologia}

Foi desenvolvida uma pesquisa descritiva com a participação de quinze profissionais de saúde que atuam na unidade oncológica do Hospital Municipal São José, em Joinville/SC.

O Hospital Municipal São José é uma entidade autárquica e constitui-se em um Centro de Referência de Alta Complexidade em Oncologia (CACON). Em 2015 realizou 41.188 atendimentos em oncologia, dos quais 5.015 em radioterapia e 20.321 de apoio a pacientes oncológicos. A Unidade de internação onde foi realizada a pesquisa possui 25 leitos e 26 profissionais trabalham no setor. O hospital atinge uma população de mais de um milhão de habitantes, compreendendo Joinville, todo o norte catarinense e cidades da microrregiāo?

Foram convidados a participar da pesquisa dezenove trabalhadores do setor de oncologia que atuavam nos quatro turnos de trabalho. Porém, quatro profissionais foram excluídos: três por se recusarem a participar da investigação e um por ter se afastado do trabalho no período em que se desenvolveu o estudo. Dessa forma, o número de entrevistados totalizou quinze profissionais 
entre enfermeiros, técnicos e auxiliares de enfermagem. A razão pela qual escolhemos esses trabalhadores deve-se ao fato de que eles permanecem a maior parte de sua jornada de trabalho na unidade oncológica e, portanto, maior tempo em contato com os pacientes oncológicos.

A Resoluçáo 466/12 do Conselho Nacional de Saúde (CNS), que trata das Diretrizes e Normas Regulamentadoras de Pesquisas envolvendo Seres Humanos, norteou a coleta de dados ${ }^{10}$. Esta pesquisa foi submetida ao comitê de ética e aprovada sob o protocolo no 1552338 .

Aqueles que concordaram em participar da investigaçáo assinaram um Termo de Consentimento Livre e Esclarecido (TCLE). O convite aos profissionais ocorreu durante as passagens de plantáo de cada turno, e na ocasiāo foram explicados os objetivos da pesquisa, seus critérios científicos e a garantia do anonimato. A coleta de dados aconteceu sempre ao final dos plantóes, entre os meses de junho e julho de 2016.

Utilizou-se como técnica de coleta de dados um questionário com perguntas abertas e fechadas especialmente desenvolvido com o objetivo de conhecer as características dos profissionais em estudo e colher informaçóes referentes a sinais e sintomas emocionais de ansiedade, depressão e estresse entre os trabalhadores investigados. Para tal, o questionário abordou os seguintes campos: condições socioeconômicas (sexo, idade, escolaridade, estado civil, quanto tempo trabalha no setor de oncologia, função, horário de trabalho, e se possui outro emprego). Em relação às condições de trabalho, foram feitas as seguintes perguntas: Existem problemas que lhe incomodam ou prejudicam a realização das suas atividades de trabalho? (sim, não, qual); Você teve algum problema de saúde nos últimos seis meses devido ao seu trabalho (sim, não, qual); Precisou ficar afastado? (sim, não); No momento, em relação a sua saúde, você tem alguma queixa que pode ter sido causada pelo trabalho? (sim, não, qual). Quanto aos sinais e sintomas emocionais, foi utilizado para a coleta de informaçóes o Self-report Questionnaire (SRQ-20). O SRQ -20 foi desenvolvido pela Organização Mundial de Saúde (OMS) com o objetivo de avaliar os transtornos mentais comuns em países em desenvolvimento. Trata-se de um questionário de identificação de distúrbios psiquiátricos em nível de atenção primária ${ }^{11}$.

O SRQ é composto por vinte questóes elaboradas para detecção de distúrbios atualmente chamados de transtornos mentais comuns (TMC), e vem sendo utilizado em vários países de diferentes culturas para o rastreamento de transtornos não psicóticos como insônia, fadiga, irritabilidade, esquecimento, dificuldade de concentração e queixas somáticas ${ }^{12}$.

Dessas questões, quatro abordam sintomas físicos e dezesseis sintomas psicoemocionais que são separados em grupos, assim denominados: humor depressivo-ansioso (pergunta 1 a 4); assusta-se com facilidade? Sentese nervoso(a), tenso(a) ou preocupado(a)? Tem se sentido triste ultimamente? Tem chorado mais do que de costume?; Sintomas somáticos (perguntas 5 a 10); Tem dores de cabeça frequentes? Tem falta de apetite? Dorme mal? Tem má digestão? Tem sensaçôes desagradáveis no estômago? Tem tremores nas mãos?; Decréscimo de energia vital (pergunta 11 a 16); Tem dificuldade para pensar com clareza? Tem dificuldades para tomar decisões? Cansa-se com facilidade? Sente-se cansado(a) o tempo todo? Tem dificuldades no serviço (seu trabalho é penoso, causa sofrimento)? Encontra dificuldades para realizar com satisfação suas atividades diárias? Pensamentos depressivos (pergunta 17 a 20); É incapaz de desempenhar um papel útil em sua vida? Tem perdido o interesse pelas coisas? Sente-se uma pessoa inútil, sem préstimo? Tem tido ideias de acabar com a vida?

Por fim, o trabalhador foi questionado quanto a sentimentos frente à situação de ter que lidar com o paciente com câncer (se o profissional percebe ter algum sentimento e/ou comportamento diferenciado quando atende pacientes com câncer).

As respostas foram organizadas no programa Excel, em planilhas desenhadas para o estudo. As variáveis pertinentes às condiçóes socioeconômicas foram calculadas por meio de percentual. Neste estudo categorizaram-se as respostas que emergiram das perguntas abertas relacionadas aos problemas de saúde, medicamentos utilizados pelos entrevistados, condiçóes de trabalho e sentimentos do trabalhador frente à situação de ter que lidar com o paciente com câncer.

As categorias de análise têm uma conotação classificatória que surge a partir do recorte do texto em unidades de registro que podem ser palavras, expressóes, frases ou acontecimentos. Para efeito de constituiçáo das categorias poderá ser considerada a repetição de conteúdos comuns à maioria dos respondentes, ou expressóes que não se repetem no relato de outros respondentes, mas que guardam em si riqueza e relevância para o estudo ${ }^{13}$.

Duas categorias foram consideradas para os problemas de saúde: distúrbio do aparelho osteoarticular e distúrbios mentais e emocionais. Quanto aos medicamentos, utilizaram-se três categorias: anti-inflamatórios e analgésicos; antidepressivos e anti-estressantes; e medicamentos cardiovasculares. Ao final, os resultados brutos dessas categorias, a exemplo das respostas as perguntas das condiçôes socioeconômicas, também foram submetidos à operação estatística simples (percentual).

Em relação às respostas às perguntas abertas sobre as condiçōes de saúde, adotaram-se oito categorias: falta de leitos, medicamentos e materiais de consumo, gestão, estrutura física inadequada, absenteísmo, clientela e familiares e inexistência de apoio psicológico aos 
trabalhadores. Quanto aos sentimentos do trabalhador frente à situação de ter que lidar com o paciente com câncer, utilizou-se como categoria de análise frustação, compaixão, tristeza, empatia e impotência.

Sobre os sinais e sintomas emocionais de ansiedade, depressão e estresse entre os trabalhadores, o SRQ20 considera como possível caso a pessoa que obtiver a pontuação de sete ou mais respostas afirmativas, que valem um ponto cada uma. Esse escore foi obtido pela determinação da sensibilidade, especificidade e dos valores preditivos positivos e negativos em outros estudos. Esse ponto de corte permite a obtenção de dois grupos: de um lado os indivíduos com maior probabilidade de ter um transtorno mental comum, e de outro um grupo com maior probabilidade de náo ter ${ }^{11}$.

$\mathrm{Na}$ análise dos achados foram consideradas as evidências científicas, e essa etapa incluiu a comparação entre os resultados encontrados e as informações presentes na literatura. Esse procedimento visou identificar discordâncias com os achados ou similaridades apesar das diferentes circunstâncias. Dada comparação possibilitou, portanto, a confrontação da realidade a visóes teóricas.

\section{Resultados e discussão}

\section{Características socioeconômicas dos profissionais}

Em relação aos profissionais, foram consideradas as variáveis sexo, idade, estado civil, escolaridade, função ocupada, tempo trabalhado no setor, carga horária e duplo vínculo de trabalho.

A idade dos participantes variou entre 27 e 58 anos; $40 \%$ encontrava-se na faixa etária entre 27 e 37 anos; $34 \%$ na de 39 a 49 anos; e $26 \%$ na de 50 a 58 anos. A média de idade foi de 41,46 anos $( \pm 9,86)$. A comparação das médias de idade dos profissionais, encontradas em outras investigaçóes, mostrou que a média de idade dos profissionais da unidade oncológica em Joinville é maior do que as observadas em um hospital oncológico no município de Uberaba/MG, e no serviço de quimioterapia de um município do Rio Grande do Sul, onde as médias foram, respectivamente, de 32,5 e 33,3 anos ${ }^{14,15}$.

A maioria (73\%) era do sexo feminino, demonstrando a predominância das mulheres na prestação de cuidados em saúde. A participaçáo das mulheres no mercado de trabalho em saúde vem sendo estudada há algumas décadas, mostrando sua importância não só para entender a expansão da participação feminina no mercado de trabalho, como para compreender as características do setor de saúde, responsável por um contingente expressivo de postos de trabalho ocupados por mulheres ${ }^{16-18}$.

Quanto à escolaridade, sobressaíram-se aqueles que possuíam qualificação mais avançada; $40 \%$ dos profissionais com terceiro grau completo e $20 \%$ com terceiro grau incompleto. Entretanto, apenas três (20\%) eram enfermeiros e grande parte dos entrevistados, 12 $(80 \%)$ eram técnicos ou auxiliares de enfermagem, para os quais se exige apenas o $2^{\circ}$ grau. A pesquisa sobre o perfil da enfermagem no Brasil realizada em 2015 pelo Conselho Federal de Enfermagem (Cofen) em parceria com a Escola Nacional de Saúde Pública (ENSP) corrobora os dados citados acima, que apontam os trabalhadores de nível médio (técnicos e auxiliares) com escolaridade acima da exigida para o desempenho de suas atribuiçōes, com $23,8 \%$ reportando nível superior incompleto e $11,7 \%$ tendo concluído o curso superior $^{19}$. Tal situaçấo sugere que esses profissionais estejam empenhados em melhorar sua remuneração ou mesmo sua posição social. Em relação ao estado civil, a maior parte desses profissionais eram casados.

Quanto ao tempo de trabalho no setor, duas profissionais tinham menos de um ano, dez profissionais tinham de um a menos de cinco anos, e três, cinco anos e mais. Esses resultados demonstram que o tempo de permanência no setor é baixo, sugerindo uma rotatividade considerável entre os profissionais, pois $80 \%$ trabalhavam a menos de cinco anos no setor oncológico. Outros dois estudos apontam para a mesma tendência, que demonstra a preponderância de elevada rotatividade entre os profissionais que atuam em áreas oncológicas ${ }^{20,21}$. A rotatividade elevada pode ser considerada como um fator desfavorável para a melhoria da qualidade da assistência, visto que o desenvolvimento do cuidado passa a ser realizado por profissionais com menor experiência, além disso, sugere uma incompatibilidade com a proposta e as condiçôes de trabalho da instituição.

A respeito do duplo vínculo de trabalho, $87 \%$ dos entrevistados negaram possuir outro emprego, o que de certa maneira é um aspecto positivo e importante. Percebe-se que um percentual menor de 13\% tem outro vínculo formal de trabalho. Esse dado diverge do estudo de Felli ${ }^{22}$ realizado em um contexto nacional, que apurou múltiplos vínculos entre os profissionais de enfermagem em razão da baixa remuneração.

Estudos demonstram que a múltiplos vínculos de trabalho influem diretamente na saúde do profissional, pois ao comprometer suas horas de repouso pode esgotar-se emocional e fisicamente ${ }^{23,24}$.

As duplas jornadas podem gerar conflitos nas relações de trabalho, pois quando os profissionais estão cansados, geralmente acabam priorizando uma parte do serviço em detrimento de outras atividades também necessárias, podendo ocasionar atrito nas relações de 
trabalho e o comprometimento da assistência de qualidade. Além disso, as duplas jornadas de trabalho podem fazer que muitos fiquem estressados, doentes e acabem faltando ao serviço ${ }^{25}$.

No que tange aos turnos, $73 \%$ dos participantes trabalham no turno diurno, e $23 \%$ trabalham em plantóes noturnos. O turno diurno, como esperado, foi o horário de trabalho mais referido entre os entrevistados. No hospital estudado, esse turno de trabalho detém o maior quantitativo de pessoal, em funçâo da maior demanda de trabalho.

\section{Situação de trabalho e problemas de saúde referidos pelos profissionais}

As questôes abordadas a seguir tratam de investigar a situação de trabalho e os problemas de saúde referidos pelos profissionais. Quando interrogados sobre a existência de problemas que incomodavam ou prejudicavam a realização das atividades diárias de trabalho, a maioria (67\%) respondeu positivamente. Os problemas relativos às condiçóes de trabalho foram apontados com maior frequência e mostram as dificuldades que os profissionais têm em lidar com falta de leitos, medicamentos e materiais de consumo, número reduzido de profissionais, estrutura física inadequada, absenteísmo, clientela e familiares, e inexistência de apoio psicológico aos trabalhadores.

De acordo com o relato de alguns profissionais:

"Geralmente problemas relacionados à gestão prejudicam, com relação a materiais que faltam, leitos ruins, falta de medicaçóes... isso sim incomoda.”(P2)

Ou ainda:

"A estrutura fisica do ambiente, falta de medicamentos de trabalho." (P3)

Outro profissional evidencia os problemas relacionados às condiçóes de trabalho, como no relato a seguir:

"O ambiente estrutural tem mais de 100 anos, bancadas velhas comidas de cupim, camas e criados-mudos enferrujados e com defeitos para o manuseio." (P6)

As condiçôes de trabalho são influenciadas e determinadas por vários fatores de ordem econômica, técnica e organizacional, que se inter-relacionam, constituindo o meio e a forma em que se desenvolve a atividade de trabalho. Antunes e Costa ${ }^{26}$ referem que nas instituiçôes públicas vêm se evidenciando que as precárias condiçōes de trabalho são desfavoráveis à saúde dos profissionais.
Os autores confirmam que a crise no sistema de saúde brasileiro, principalmente nas organizaçóes hospitalares públicas, está associada à conjuntura econômica do país, citam que a gravidade desse problema aumentou após a reforma do Estado, tendo em vista que esta definiu como diretrizes básicas a redução de despesas, principalmente do quantitativo de funcionários públicos, propiciando, dentre outros, efeito imediato na política de recursos humanos em saúde, afetando diretamente o quantitativo de pessoal nas instituiçóes hospitalares.

Para Mauro, Braga e $\mathrm{Lima}^{27}$ a assistência à saúde tornou-se cada vez mais complexa, exigindo mais equipamentos, profissionais mais capacitados, o que leva à consequente elevaçáo dos custos dos procedimentos. Em contraponto, há a redução das receitas, corte dos recursos humanos, que provocam a sobrecarga das atividades dos profissionais de saúde. O estudo de Spindola e Santos ${ }^{28}$ corrobora esse entendimento ao afirmar que a assistência de enfermagem nas instituiçóes públicas tem sido penalizada com a deficiência dos recursos humanos e materiais, o que interfere diretamente na qualidade da assistência prestada à população, gerando insatisfação nos profissionais que se sentem impotentes e frustrados com a situação.

Ao realizarem uma pesquisa bibliográfica, outros autores identificaram vários estudos que descrevem as condiçóes de trabalho como um dos fatores desencadeantes de doenças ocupacionais entre os profissionais que atuam no setor de saúde ${ }^{29}$.

O absenteísmo é outra situação preocupante apontada pelos trabalhadores da oncologia, de acordo com os relatos:

"Falta de funcionários e falta de medicamentos."(P2)

"Falta de funcionários. complexidade dos pacientes."(P7)

O absenteísmo é um importante indicador de avaliação da saúde dos trabalhadores e das condiçóes em que o trabalho é realizado, como também da política de recursos humanos da instituição ${ }^{30}$.

De acordo com Bezerra ${ }^{31}$, o absenteísmo tem consequências à queda na qualidade da assistência, sobrecarga de atividades, falta de motivaçáo do profissional ativo, risco na saúde do trabalhador comprometido e aumento dos custos financeiros despendidos com horas extras.

Pesquisas realizadas com trabalhadores da enfermagem apontam para a diversidade e gravidade dos problemas de saúde dos profissionais, que apresentam desgastes biopsíquicos em virtude da própria natureza do trabalho e pelas condiçóes em que o mesmo é realizado ${ }^{32-34}$.

Outros estudos chamam atenção para a alta frequência de ausências, motivadas por licenças-médicas, em diferentes unidades hospitalares e determinadas por 
doenças do sistema osteomuscular e tecido conjuntivo, e pelos transtornos mentais. Esses tipos de problemas representaram os dois grupos de doenças com maior impacto na quantidade de dias de licença ${ }^{35,36}$. No caso do setor de oncologia em Joinville, $40 \%$ dos entrevistados referiram problemas osteomusculares.

Outro ponto a ser discutido no presente estudo resulta do relato dos profissionais que expóem sua dificuldade para lidar com problemas durante a convivência diária junto aos familiares dos pacientes. Trechos de entrevistas feitas com vários profissionais demonstram que os trabalhadores lidam cotidianamente com situaçōes de estresse de difícil resolução.

Conforme alguns relatos:

"A falta de apoio psicológico para pacientes e acompanhantes, que na maioria das vezes descarregam suas frustaçóes na enfermagem, que também trabalha com número reduzido de funcionários" (P.3),

"Falta de estrutura física, humana, psicológica, sinto que o setor está em total abandono." (P5)

E ainda:

"Acompanhantes deveriam ser mais bem orientados sobre estado geral do paciente pelo médico, psicólogo ou enfermeiros, pois na oncologia, as campainhas tocam o tempo todo, os acompanhantes não saem da porta do posto de enfermagem. Falta de matérias, distribuição de tarefas, 3 horários de medicaçoes, banhos já divididos, exames a maior parte à tarde, deveria ter melhor distribuição dos afazeres por turnos." (P4)

Em consequência desses relatos, é possível sugerir que as condiçôes de trabalho no setor de oncologia parecem ser inadequadas às reais necessidades dos trabalhadores, principalmente aquelas relacionadas à falta de recursos humanos e materiais para a prestação da assistência aos pacientes. Tais condiçóes aumentam o sofrimento mental e físico dos profissionais, pois compete a eles, além de fazer adaptaçóes no processo de trabalho, quase sempre em condiçôes precárias, ter que mobilizar forte carga afetiva para dar suporte ao paciente e à família.

Cabe aqui salientar outro aspecto identificado neste estudo, e que se assemelha com a literatura quando discorre sobre os sentimentos de frustaçáo, compaixáo e tristeza dos profissionais frente ao paciente oncológi$\mathrm{Co}^{37}$. Sobre isso, as falas dos profissionais entrevistados expressam essas perspectivas:

"Sinto pena ao ver o sofrimento e tento desempenhar um papel que traga conforto e alivio para dor deles." (P14)

“Sinto pena, é o pior lugar do hospital para se trabalhar."(P8)
"Comoção, incapacidade e tristeza." (P3)

Os sentimentos, como pena e compaixão, estão presentes no cotidiano da enfermagem e refletem a humanização, no sentido de solidariedade, presentes na assistência. A compaixáo é compreendida como uma ação humanística e de empatia, de forma que há uma identificação. Cuidar, do cliente que está padecendo, fragiliza, assusta e traz muita tristeza ${ }^{38}$. Já a tristeza pode estar relacionada à impotência dos profissionais diante da doença.

Os relatos a seguir são ilustrativos quanto a esse sentimento:

"A sensação que lidamos diariamente é de impotência. Trabalhamos incessantemente, mas os frutos (resultados) são poucos." (P11)

"Na maioria das vezes tenho a sensação que todo o trabalho com os pacientes oncológicos é inútil, com exceção dos procedimentos que proporcionam conforto, uma vez que a maioria dos casos termina em óbito." (P6)

A impotência relacionada ao paciente terminal está na sensação de insuficiência, na espera da morte. A desesperança nas medidas terapêuticas disponíveis geram um tipo de paralisia frente às situaçóes e as demandas. Comportamento decorrente da angústia pelo discernimento de que o câncer pode ser capaz de levar à morte, independente das medidas e esforços que são tomados ${ }^{39}$. O estigma da morte institui um vazio difícil de compreender, que é dolorido e faz sofrer. Quando este vazio se une a algo que remete à natureza finita e limitada do ser, confrontado com a iminência do tempo, que é restrito, indica a fragilidade do ser humano. Diante dessas indagaçōes, os trabalhadores de saúde defrontam-se permanentemente com uma ocorrência fundamental para se compreender o ser humano: a morte, sensação que causa angústia ${ }^{2}$.

Por fim, foi constatada nas falas dos profissionais a empatia:

"Acredito que devemos entender a forma que às vezes somos tratados pelos pacientes devido sua condiçâo, e que às vezes somos seus pontos de gatilho, tratar com respeito, carinho e ao mesmo tempo saber não absorver tudo de ruim que vivenciamos". (P12)

Foi constatado também o sentimento de realização, de dever cumprido e satisfação, expressos nos relatos que seguem:

"Dar o melhor conforto possivel dentro das condiçôes de trabalho e ciente que não podemos fazer milagres. Dar o conforto na enfermidade e na fase final da vida." (P10) 
"Tento fazer o máximo para suavizar o sofrimento"(P14)

Uma pesquisa realizada com profissionais de enfermagem em uma ala oncológica de um hospital no estado do Paraná confirma o pensamento dos entrevistados desse estudo, quando relatam que para cuidar de um doente fora de possibilidade terapêutica, o profissional precisa ter empatia, respeito e sensibilidade.

Para Kessler e Krug ${ }^{40}$ a enfermagem que presta assistência ao paciente oncológico convive com um turbilhão de sentimentos, desde o sofrimento até a satisfação profissional. Conforme Kovacs ${ }^{41}$ a equipe de enfermagem tem alto risco de colapso pela sua função de cuidado diário aos pacientes com dor e sofrimento, e em razão da coexistência com sentimentos contraditórios, que os colocam em conflito permanente.

Santos ${ }^{42}$ diz que ajudar outras pessoas sempre foi reconhecido como uma ação nobre, porém os custos emocionais desta ação só têm tido atenção recentemente. O exercício de profissóes como a Enfermagem implica uma relaçáo permeada por ambiguidade, como conviver com a distinção entre envolver-se somente como profissional e não como pessoa no ato de ajudar o próximo.

A partir desse momento serão mostrados os resultados obtidos por meio das respostas dos entrevistados segundo os problemas de saúde referidos, afastamentos do trabalho, queixas relacionadas ao trabalho e tipo de medicamento consumido (Tabela 1).

Tabela 1: Distribuição dos profissionais segundo problema de saúde referido, afastamentos do trabalho, respostas a queixas relacionadas ao trabalho e tipo de medicamento consumido.

\begin{tabular}{lc} 
Variáveis/categorias & N (\%) \\
Problemas de saúde recentes & $1(7)$ \\
Distúrbios mentais e emocionais & $6(40)$ \\
Distúrbio do aparelho osteoarticular & $8(53)$ \\
Nenhum problema referido & \\
Afastamento do trabalho & $4(27)$ \\
Sim & $11(73)$ \\
Não & \\
Queixas relacionadas ao trabalho & $2(13)$ \\
Sobrecarga de trabalho & $5(33)$ \\
Dor & $2(13)$ \\
Cansaço físico e mental & $6(40)$ \\
Sem queixas & \\
Utilizaçáo frequente de & \\
medicamentos & $1(7)$ \\
Cardiovasculares & $3(20)$ \\
Anti-inflamatórios e analgésicos & $1(7)$ \\
Antidepressivos e antiestressantes & $10(67)$ \\
Não fazem uso de medicaçáo & \\
\hline
\end{tabular}

Legenda: $\mathrm{N}=$ população total. \% frequência

Os resultados da tabela 1 indicam que o grupo de doenças do sistema osteomuscular representa $40 \%$ dos problemas de saúde referidos pelos entrevistados. Quanto à prevalência de dor, este estudo constatou que $33 \%$ de trabalhadores de enfermagem referiram dor nos últimos seis meses, e os medicamentos mais utilizados foram os analgésicos e anti-inflamatórios (20\%). A comparação dos resultados dessa investigaçáo com outras duas pesquisas mostrou que o percentual de doença osteomuscular observada nos profissionais de enfermagem investigados foi maior do que as observadas em uma instituiçáo pública de saúde do interior do estado de São Paulo e na Fundação Hospitalar Estadual de Minas Gerais, em Belo Horizonte, onde os percentuais de distúrbios músculo-esqueléticos entre os profissionais de saúde foram de $31,9 \%$ e $11,83 \%$, respectivamente ${ }^{43,44}$.

Os problemas osteomusculares referidos podem justificar o uso de analgésicos e anti-inflamatórios como principal medicamento utilizado pelos entrevistados. Estudos recentes confirmam a maior frequência de doenças osteomusculares entre os trabalhadores de enfermagem ${ }^{45,46}$.

A predominância das cargas fisiológicas, evidenciadas pela manipulaçáo de peso e adoção de posturas inadequadas na movimentação de pacientes, são apontadas como geradores de problemas osteomusculares e do tecido conjuntivo nos trabalhadores de enfermagem, incidindo em afastamento ao trabalho ${ }^{46}$.

Em relação às doenças osteomusculares, a Organização Mundial da Saúde estimou que, para o ano de 2015 esse tipo de enfermidade seria a maior causa de gastos em saúde, considerando que se constitui em uma das causas mais frequentes de absenteísmo laboral e invalidez permanente.

Para Gurgueira, Alexandre e Corrêa ${ }^{47}$ a dor é uma das principais queixas físicas, sendo as musculares a principal causa de dor crônica em toda a sociedade. As lombalgias e dores generalizadas vêm sendo descritas cada vez mais. Esses sintomas apresentam impacto significativo na qualidade de vida das pessoas.

\section{Análise dos casos suspeitos de TMC segundo respostas do SRQ-20}

A seguir serão apresentados casos suspeitos de TMC entre os entrevistados, que foram identificados por meio da aplicação do instrumento de rastreamento SRQ-20.

A partir da aplicação do questionário SRQ-20, e considerando a nota de corte $\geq 7$ para a suspeita de TMC, identificou-se que $20 \%$ dos trabalhadores do setor de oncologia do HMSJ apresentaram rastreamento positivo para TMC (Quadro 1).

A comparação dos percentuais dos casos suspeitos de TMC encontrados em outros três estudos mostra que a percentualidade do setor de oncologia em Joinville é superior a observada em um estudo realizado com profissionais de enfermagem de um hospital especializado de 
pequeno porte no município de Feira de Santana, no interior do Estado da Bahia, que identificou um percentual de $14,8 \%$ de casos suspeitos de TMC entre os trabalhadores. Entretanto, é inferior a encontrada em um estudo realizado entre profissionais da Estratégia de Saúde da Família de um município do interior do Rio Grande do Sul, que identificou $34 \%{ }^{48}$ de casos suspeitos de TMC. Contudo, se aproximou do percentual $(21,7 \%)$ observado por Lima ${ }^{49}$ em um estudo na população urbana de Botucatu/SP.

No grupo de profissionais identificados como casos positivos, as questóes do SRQ-20 com resposta mais frequente $(100 \%)$ foram as que se referiram ao nervosismo, tensão ou preocupação inserida no grupo de perguntas que avaliam humor ansioso.
$\mathrm{Na}$ avaliação referente aos sintomas somáticos, aparecem com $100 \%$ das respostas as sensaçóes desagradáveis no estomago e a má digestão. Quanto ao decréscimo de energia vital, a presença do cansaço foi relatada por todos os trabalhadores com suspeita de TMC. Sobre o grupo de sintomas que avaliam pensamentos depressivos, a resposta mais mencionada foi a que se refere à perda do interesse pelas coisas, com 67\% (Quadro 1).

Aqui, é necessário observar que mesmo que esses indícios, num primeiro momento, não se traduzam em doenças, e sim em sintomas, é possível inferir que eles vêm comprometendo a qualidade de vida dos trabalhadores do setor de oncologia.

Quadro 1: Distribuição dos trabalhadores do setor de oncologia do HMSJ que apresentaram rastreamento positivo para TMC de acordo com o questionário SRQ-20

\begin{tabular}{|c|c|c|c|c|c|c|c|c|c|c|c|c|c|c|c|}
\hline \multirow[t]{2}{*}{ Perguntas } & \multicolumn{15}{|c|}{ Respostas dos entrevistados } \\
\hline & 1 & 2 & 3 & 4 & 5 & 6 & 7 & 8 & 9 & 10 & 11 & 12 & 13 & 14 & 15 \\
\hline Tem tido dores de cabeça & $\mathrm{N}$ & $S$ & S & $S$ & S & S & $\mathrm{N}$ & $\mathrm{N}$ & S & $\mathrm{N}$ & $\mathrm{N}$ & $\mathrm{N}$ & $\mathrm{N}$ & $\mathrm{N}$ & $\mathrm{N}$ \\
\hline Tem falta de apetite & $\mathrm{N}$ & $\mathrm{N}$ & S & $\mathrm{N}$ & $\mathrm{N}$ & $\mathrm{N}$ & $\mathrm{N}$ & $\mathrm{N}$ & $\mathrm{N}$ & $\mathrm{N}$ & $\mathrm{N}$ & $\mathrm{N}$ & $\mathrm{N}$ & $S$ & $\mathrm{~N}$ \\
\hline Dorme mal & S & $S$ & $\mathrm{~N}$ & $\mathrm{~N}$ & $\mathrm{~N}$ & S & $\mathrm{N}$ & $\mathrm{N}$ & $\mathrm{N}$ & $\mathrm{N}$ & $\mathrm{N}$ & $\mathrm{N}$ & $\mathrm{N}$ & S & $\mathrm{N}$ \\
\hline Assusta-se com facilidade & $\mathrm{N}$ & $\mathrm{N}$ & $\mathrm{N}$ & $\mathrm{N}$ & $\mathrm{N}$ & $\mathrm{N}$ & $\mathrm{N}$ & S & $\mathrm{N}$ & $\mathrm{N}$ & $\mathrm{N}$ & S & $\mathrm{N}$ & $\mathrm{N}$ & $\mathrm{N}$ \\
\hline Tem tremores de mão & $\mathrm{N}$ & $\mathrm{N}$ & $\mathrm{N}$ & $\mathrm{N}$ & $\mathrm{N}$ & S & $\mathrm{N}$ & $\mathrm{N}$ & S & $\mathrm{N}$ & $\mathrm{N}$ & $\mathrm{N}$ & $\mathrm{N}$ & $\mathrm{N}$ & $\mathrm{N}$ \\
\hline Sente-se nervoso ou preocupado & $S$ & $\mathrm{~N}$ & $\mathrm{~N}$ & $\mathrm{~N}$ & $S$ & S & $\mathrm{N}$ & $\mathrm{N}$ & S & $\mathrm{N}$ & $S$ & $S$ & $\mathrm{~N}$ & $S$ & $\mathrm{~N}$ \\
\hline Tem má digestão & $S$ & $\mathrm{~N}$ & $\mathrm{~N}$ & $\mathrm{~N}$ & S & S & $\mathrm{N}$ & $\mathrm{N}$ & S & $\mathrm{N}$ & $\mathrm{N}$ & $\mathrm{N}$ & $\mathrm{N}$ & $S$ & $\mathrm{~N}$ \\
\hline Dificuldade para pensar com clareza & $\mathrm{N}$ & $\mathrm{N}$ & $\mathrm{N}$ & $S$ & $\mathrm{~N}$ & $\mathrm{~N}$ & $\mathrm{~N}$ & $\mathrm{~N}$ & S & $\mathrm{N}$ & $\mathrm{N}$ & $\mathrm{N}$ & $\mathrm{N}$ & $S$ & $\mathrm{~N}$ \\
\hline Tem se sentido triste & $\mathrm{N}$ & $\mathrm{N}$ & $\mathrm{S}$ & $\mathrm{N}$ & $\mathrm{N}$ & $\mathrm{N}$ & $\mathrm{N}$ & $\mathrm{N}$ & $\mathrm{S}$ & $\mathrm{N}$ & $S$ & $\mathrm{~N}$ & $\mathrm{~N}$ & S & $\mathrm{N}$ \\
\hline Tem chorado mais do que de costume & $\mathrm{N}$ & $\mathrm{N}$ & $\mathrm{N}$ & $\mathrm{N}$ & $\mathrm{N}$ & $\mathrm{N}$ & $\mathrm{N}$ & $\mathrm{N}$ & $\mathrm{N}$ & $\mathrm{N}$ & $\mathrm{N}$ & $\mathrm{N}$ & $\mathrm{N}$ & $\mathrm{N}$ & $\mathrm{N}$ \\
\hline $\begin{array}{l}\text { Encontra dificuldade para realizar com satisfação suas } \\
\text { atividades diárias? }\end{array}$ & $\mathrm{N}$ & $\mathrm{N}$ & $S$ & $\mathrm{~N}$ & $\mathrm{~N}$ & $\mathrm{~N}$ & $\mathrm{~N}$ & $\mathrm{~N}$ & $\mathrm{~N}$ & $\mathrm{~N}$ & $\mathrm{~N}$ & $\mathrm{~N}$ & $\mathrm{~N}$ & $S$ & $\mathrm{~N}$ \\
\hline Tem dificuldade para tomar decisóes & $\mathrm{N}$ & $\mathrm{N}$ & $\mathrm{N}$ & $\mathrm{N}$ & $\mathrm{N}$ & $\mathrm{N}$ & $\mathrm{N}$ & $\mathrm{N}$ & $\mathrm{N}$ & $\mathrm{N}$ & $\mathrm{N}$ & $\mathrm{N}$ & $\mathrm{N}$ & $S$ & $\mathrm{~N}$ \\
\hline $\begin{array}{l}\text { Tem dificuldade no serviço (seu trabalho é penoso, } \\
\text { causa sofrimento)? }\end{array}$ & $\mathrm{N}$ & $\mathrm{N}$ & $\mathrm{N}$ & $\mathrm{N}$ & S & $\mathrm{N}$ & $\mathrm{N}$ & S & S & $\mathrm{N}$ & S & $\mathrm{N}$ & $\mathrm{N}$ & $\mathrm{N}$ & $\mathrm{N}$ \\
\hline É incapaz de desempenhar um papel útil em sua vida & $\mathrm{N}$ & $\mathrm{N}$ & $\mathrm{N}$ & $\mathrm{N}$ & $\mathrm{N}$ & $\mathrm{N}$ & $\mathrm{N}$ & $S$ & $\mathrm{~N}$ & $\mathrm{~N}$ & $\mathrm{~N}$ & $\mathrm{~N}$ & $\mathrm{~N}$ & $S$ & $\mathrm{~N}$ \\
\hline Perda de interesse pelas coisas & $\mathrm{N}$ & $\mathrm{N}$ & $\mathrm{N}$ & $\mathrm{N}$ & $\mathrm{N}$ & $\mathrm{N}$ & $\mathrm{N}$ & $\mathrm{N}$ & $\mathrm{S}$ & $\mathrm{N}$ & $\mathrm{N}$ & $\mathrm{N}$ & $\mathrm{N}$ & S & $\mathrm{N}$ \\
\hline Sente-se uma pessoa inútil & $\mathrm{N}$ & $\mathrm{N}$ & $\mathrm{N}$ & $S$ & $\mathrm{~N}$ & $\mathrm{~N}$ & $\mathrm{~N}$ & $\mathrm{~N}$ & $\mathrm{~N}$ & $\mathrm{~N}$ & $\mathrm{~N}$ & $\mathrm{~N}$ & $\mathrm{~N}$ & $S$ & $\mathrm{~N}$ \\
\hline Tem tido ideias de acabar com a própria vida & $\mathrm{N}$ & $\mathrm{N}$ & $\mathrm{N}$ & $\mathrm{N}$ & $\mathrm{N}$ & $\mathrm{N}$ & $\mathrm{N}$ & $\mathrm{N}$ & $\mathrm{N}$ & $\mathrm{N}$ & $\mathrm{N}$ & $\mathrm{N}$ & $\mathrm{N}$ & $\mathrm{N}$ & $\mathrm{N}$ \\
\hline Sente-se cansado(a) o tempo todo & $S$ & $\mathrm{~N}$ & S & $\mathrm{N}$ & $\mathrm{N}$ & $S$ & $\mathrm{~N}$ & $\mathrm{~N}$ & S & $\mathrm{N}$ & $S$ & $\mathrm{~N}$ & $\mathrm{~N}$ & $S$ & $\mathrm{~N}$ \\
\hline Tem sensações desagradáveis no estomago & S & $\mathrm{N}$ & $\mathrm{N}$ & $\mathrm{N}$ & $\mathrm{N}$ & S & $\mathrm{N}$ & $\mathrm{N}$ & $S$ & $\mathrm{~N}$ & $\mathrm{~N}$ & $\mathrm{~N}$ & $\mathrm{~N}$ & $S$ & $\mathrm{~N}$ \\
\hline
\end{tabular}


Quadro 1. Continuação.

\begin{tabular}{lccccccccccccccccc} 
Perguntas & \multicolumn{11}{c}{ Respostas dos entrevistados } \\
& $\mathbf{1}$ & $\mathbf{2}$ & $\mathbf{3}$ & $\mathbf{4}$ & $\mathbf{5}$ & $\mathbf{6}$ & $\mathbf{7}$ & $\mathbf{8}$ & $\mathbf{9}$ & $\mathbf{1 0}$ & $\mathbf{1 1}$ & $\mathbf{1 2}$ & $\mathbf{1 3}$ & $\mathbf{1 4}$ & $\mathbf{1 5}$
\end{tabular}

Legenda: S: sim; N: nâo

Fonseca, Guimarães e Vasconcelos ${ }^{50}$ nomeiam sofrimento difuso como queixas somáticas inespecíficas, citando como principais sintomas dores de cabeça e no corpo, nervosismo, problemas gástricos, cansaço e estados de mal-estar não classificáveis nos diagnósticos médicos e psiquiátricos, onde as múltiplas raízes podem ser encontradas nas relações sociais, familiares ou de trabalho.

Vale a pena lembrar as difíceis condiçôes de trabalho relatadas pelos profissionais neste estudo. Entretanto, além das condições de trabalho, há também a assistência ao paciente oncológico com suas particularidades, visto que estes são portadores de uma patologia complexa e o seu tratamento, na maioria das vezes, é muito agressivo, acarretando ao profissional não somente uma alta demanda emocional, mas também psíquica.

Seligmann-Silva et $\mathrm{al}^{51}$ consideram a existência de muitos elementos estressores no ambiente de trabalho da enfermagem, que demandam dos trabalhadores exigências físicas e mentais. Apontam a enfermagem como uma das mais afetadas por doenças do trabalho, dentre as quais são identificadas uma crescente incidência de TMC.

Para Chaves, Ramos e Figueiredo ${ }^{52}$ os profissionais de saúde vivem várias situaçóes que os expóem ao sofrimento e aparecimento de doenças, que são destacadas por sinais e sintomas orgânicos e psíquicos, como por exemplo os transtornos mentais, afetando assim o seu desempenho no trabalho e a qualidade da assistência prestada.

Análise dos grupos de sintomas do SRQ- 20 referidos pelos trabalhadores

A seguir será apresentado o resultado dos sintomas referidos por todos os trabalhadores entrevistados, num total de 15 .

Tabela 2: Distribuição dos grupos de sintomas do SRQ-20 referidos por todos os trabalhadores de enfermagem entrevistados.

\begin{tabular}{|c|c|c|}
\hline Grupo de sintomas/alteraçóes de saúde & $\mathbf{N}$ & $\%$ \\
\hline \multicolumn{3}{|l|}{ Humor depressivo-ansioso } \\
\hline Nervosismo, tensão ou preocupação & 7 & 47 \\
\hline Assusta-se com facilidade & 2 & 13 \\
\hline Sente-se triste ultimamente & 4 & 27 \\
\hline Chora mais do que o costume & - & - \\
\hline \multicolumn{3}{|l|}{ Sintomas sintomáticos } \\
\hline Dores de cabeça & 6 & 40 \\
\hline Falta de apetite & 2 & 13 \\
\hline Dorme mal & 4 & 27 \\
\hline Má digestấo & 5 & 33 \\
\hline Tremores na mão & 2 & 13 \\
\hline Desconforto estomacal & 4 & 27 \\
\hline \multicolumn{3}{|l|}{ Decréscimo de energia vital } \\
\hline Dificuldade para pensar com clareza & 3 & 20 \\
\hline Cansaço com facilidade & 5 & 33 \\
\hline Dificuldade para tomar decisões & 1 & 7 \\
\hline Dificuldade ao realizar as atividades com satisfação & 2 & 13 \\
\hline Cansaço contínuo & 6 & 40 \\
\hline Dificuldade no trabalho & 4 & 27 \\
\hline \multicolumn{3}{|l|}{ Pensamentos depressivos } \\
\hline Incapaz de desempenhar um papel útil em sua vida & 2 & 13 \\
\hline Perda de interesse pelas coisas & 2 & 13 \\
\hline Sente-se inútil & 2 & 13 \\
\hline Pensa em dar fim à vida & - & - \\
\hline
\end{tabular}


Sobre os problemas de saúde, onze entrevistados afirmaram ter alguma queixa, o que equivale à proporção de $73,34 \%$. Pode-se observar que a queixa de nervosismo, tensão ou preocupação são os sintomas com maior prevalência ( $47 \%$ ) entre os trabalhadores do setor de oncologia, seguidos dos sintomas de cansaço e dores de cabeça $(40 \%)$ e má digestão (33\%). Esses dados coincidem com as principais queixas referidas por trabalhadores de enfermagem que assistem crianças em um hospital público de São Paulo, em que a tensão e as dores de cabeça são as principais queixas referidas ${ }^{53}$.

Alguns profissionais referiram ainda lesões na coluna, hematomas, depressão e dores musculares.

$\mathrm{Na}$ literatura, os TMCs caracterizados por insônia, ansiedade, fadiga, dificuldade de concentração, nervosismo e queixas somáticas são os mais relatados e representam alto custo social e econômico, por revelarem-se incapacitantes, sendo uma das causas relevantes de aumento da demanda por serviços de saúde ${ }^{54,55}$.

As influências do trabalho sobre a saúde dos trabalhadores são conhecidas desde a antiguidade, e ao longo dos últimos três séculos cresceu progressivamente a compreensão das relaçóes entre trabalho e processo saúde-doença. Avanços em vários campos do conhecimento têm contribuído para isso, particularmente os da epidemiologia, psicologia social, ergonomia, dentre outros ${ }^{54}$.

Para Alves, Godoy e Santana ${ }^{56}$ os hospitais são marcados pela intensificação do trabalho e complexidade do serviço prestado, devido às demandas e necessidades dos pacientes, gerando assim aumento da carga de trabalho, sobrecarregando os profissionais, provocando insatisfação e tensão dos membros.

\section{Conclusão}

Os achados desse estudo corroboram com as prevalências encontradas em outras populações estudadas. As questóes do instrumento SRQ-20 com maior proporção de respostas positivas versavam sobre nervosismo, tensão ou preocupação, dores de cabeça e cansaço.

Foram encontrados os fatores que influenciam o trabalho dos profissionais de enfermagem no setor de oncologia, e esses fatores podem ser descritos como aqueles relacionados às condiçóes de trabalho, dificuldade em lidar com a terminalidade do paciente oncológico e a relação com familiares.

Além disso, evidenciam-se a prevalência de TMC entre esses trabalhadores. Tais resultados indicam necessidade de intervenção, visando melhorar as condiçôes gerais de trabalho e fornecer suporte emocional ao coletivo de trabalhadores.
Por fim, duas limitações podem ser apontadas neste estudo. A primeira, que essa investigação possui uma amostra pequena quando comparada à população total de profissionais de enfermagem na instituiçáo em estudo. E é fato que o resultado desse estudo náo pode ser extrapolado para os demais profissionais. A segunda, é que o SRQ-20, apesar de sua alta sensibilidade, é um instrumento de rastreamento e não de diagnóstico. Entretanto, é um recurso que possibilita a apreensão de determinada uma manifestação de sofrimento.

\section{Referências}

1. Brasil. Ministério da Saúde. Instituto Nacional do Câncer. Câncer matou 8,2 milhôes de pessoas em 2012 [Internet]. c2013 [citado em 2017 mar 27]. Disponível em: http://bit. ly/2nsMpF0

2. Silva LC. O sofrimento psicológico dos profissionais de saúde na atenção ao paciente de câncer. Psicol Am Lat [Internet]. 2009 [citado em 2016 out 25];(16). Disponível em: http://bit.ly/2mZeG2F

3. Hercos TM, Vieira FS, Oliveira MS, Buetto LS, Shimura CMN, Sonobe HM. O trabalho dos profissionais de enfermagem em unidades de terapia intensiva na assistência ao paciente oncológico. Rev Bras Cancerologia [Internet]. 2014 [citado em 2016 abr 12];60(1):51-8. Disponível em: http://bit.ly/2nNoDnX

4. Fernandes PV, Iglesias A, Avellar LZ. O técnico de enfermagem diante da morte: concepçóes de morte para técnicos de enfermagem em oncologia e suas implicações na rotina de trabalho e na vida cotidiana. Psicol Teor Prat [Internet]. 2009 [citado em 2016 out 13];11(1):142-52. Disponível em: http://bit.ly/2nNsRMc

5. Oliveira ECN. O psicólogo na UTI: reflexôes sobre a saúde, vida e morte nossa de cada dia. Psicol Cienc Prof [Internet]. 2002 [citado em 2016 ago 10];22(2):30-41. Disponível em: http://bit.ly/2nWdNZI

6. Porto AR, Thofehrn DDP, Amestoy SC, Arrieira ICO, Jone LR. Visão dos profissionais sobre seu trabalho no programa de internação domiciliar interdisciplinar oncológico: uma realidade brasileira. Av Enferm [Internet]. 2014 [citado em 2016 mar 1];32(1):72-9. Disponível em: http://bit. ly/2nIwK4Q

7. Franco MHP. Cuidados paliativos e o luto no contexto hospitalar. Mundo Saúde. 2003;27(1):182-4.

8. Faria DAP, Maia EMC. Ansiedades e sentimentos de profissionais da enfermagem nas situações de terminalidade em oncologia. Rev Latino-Am Enfermagem [Internet]. 2007 [citado em 2016 set 12];15(6):1131-7. Disponível em: http://bit.ly/2nPtwtU

9. Joinville. Hospital Municipal São José. Histórico [Internet]. 2016 [citado em 2016 jan]. Disponível em: http://www. hmsj.sc.gov.br/ 
10. Brasil. Conselho Nacional de Saúde. Resolução no 466, de 12 de dezembro de 2012. Diretrizes e normas regulamentadoras de pesquisas envolvendo seres humanos. Diário Oficial da União. Brasília, DF; 13 jun. 2012.

11. Santos KOB, Araújo TM, Oliveira NF. Estrutura fatorial e consistência interna do Self-Reporting Questionnaire (SRQ20) em população urbana. Cad Saúde Públ [Internet]. 2009 [citado em 2016 set 12];25(1):214-22. Disponível em: http://bit.ly/2mVNkcT

12. Gonçalves DM, Stein AT, Kapczinski F. Avaliação de desempenho do self-reporting questionnaire como instrumento de rastreamento psiquiátrico: um estudo comparativo com o Structured Clinical Interview for DSM-IV-TR. Cad Saúde Pública [Internet]. 2008 [citado em 2016 ago 14];24(2):380-90. Disponível em: http://bit.ly/2oa2X1o

13. Minayo MCS. Ciência técnica e arte: o desafio da pesquisa social [Internet]. 2009 [citado em 2016 ago 12]. Disponível em: http://www.densf.xpg.com.br/modulo1.htm

14. Faquim ACM. Qualidade do cuidado e contexto de trabalho do profissional de enfermagem em um hospital oncológico [dissertação]. Uberaba: Universidade Federal do Triângulo Mineiro; 2014.

15. Silva BQ, Hahn SR. Uso de plantas medicinais por indivíduos com hipertensão arterial sistêmica, diabetes mellitus ou dislipidemias. Rev Bras Farm Hosp Serv Saúde São Paulo [Internet]. 2011 [citado em 2016 jun];2(3):36-40. Disponível em: http://bit.ly/2nhTYOk

16. Lopes MJM, Leal SMC. A feminização persistente na qualificação profissional da enfermagem brasileira. Cad Pagu [Internet]. 2005 [citado em 2016 set 13];24:105-25. Disponível em: http://bit.ly/2oC4s9A

17. Brito JC, Neves MY, Oliveira SS, Rotenberg L. Saúde, subjetividade e trabalho: o enfoque clínico e de gênero. Rev Bras Saúde Ocup [Internet]. 2012 [citado em 2016 set 22];37(126):31629. Disponível em: http://bit.ly/2mZmRw3

18. Matos IB, Toassi RFC, Oliveira MC. Profissões e ocupaçôes de saúde e o processo de feminização: tendências e implicaçôes. Ath Digital [Internet]. 2013 [citado em 2016 set 24];13(2):239-44. Disponível em: http://bit.ly/2mxbyyU

19. Escola Nacional de Saúde Pública. Perfil da Enfermagem no Brasil [Internet]. 6 maio 2015 [citado em 2016 set 27]. Disponível em: http://bit.ly/2nhS3co

20. Bordignon M, Monteiro MI, Mai S, Martins MFSV, Rech CRA, Trindade LL. Satisfação e insatisfação no trabalho de profissionais de enfermagem da oncologia do Brasil e Portugal. Texto \& Contexto Enferm [Internet]. 2015 [citado em 2016 set 13];24(4):925-33. Disponível em: http://bit.ly/2nWmenB

21. Baía WRM. Rotatividade dos profissionais de enfermagem durante a fase de implantação de uma instituição de saúde de alta complexidade [tese]. São Paulo: Universidade de São Paulo; 2014.

22. Felli VEA. Implantação e avaliação do sistema de monitoramento da saúde do trabalhador de enfermagem. Relatório de pesquisa [Fapesp]. São Paulo: Escola de Enfermagem da USP; 2010.
23. Pafaro RC, De Martino MMF. Estudo do estresse do enfermeiro com dupla jornada de trabalho em um hospital de oncologia pediátrica de Campinas. Rev Esc Enferm USP [Internet]. 2004 [citado em 2016 set 23];38(2):152-60. Disponível em: http://bit.ly/2nhO2Ve

24. Cechin P, Freitas HMB, Ilha S, Martins ESR, Souza MHT. Alteraçóes vivenciadas por profissionais de enfermagem que apresentam dupla jornada de trabalho. Rev Enferm UFPE [Internet]. 2014 [citado em 2016 ago 12];8(11):3855-61. Disponível em: http://bit.ly/2nIEu6V

25. Nogueira MLG. Afastamentos por adoecimento de trabalhadores de enfermagem em oncologia [dissertação]. Rio de Janeiro: Universidade Federal do Estado do Rio de Janeiro; 2007.

26. Antunes AV, Costa MN. Dimensionamento de pessoal de enfermagem em um hospital universitário. Rev Latino-Am Enferm [Internet]. 2003 [citado em 2016 ago 13];11(6)8329. Disponível em: http://bit.ly/2nNyUke

27. Mauro CCC, Braga LB, Lima MB. Análise do processo de trabalho em uma clínica médica: a atividade da auxiliar de enfermagem. Rio de Janeiro; 2003. Mimeografado.

28. Spindola T, Santos RS. O trabalho na enfermagem e seu significado para as profissionais. Rev Bras Enferm [Internet]. 2005 [citado em 2016 set 10];58(2):156-60. Disponível em: http://bit.ly/2nWmHGJ

29. Teixeira LP, Silva TASM. Doenças ocupacionais na enfermagem: quando o trabalho adoece. Revista Pró-UniverSUS [Internet]. 2014 [citado em 2016 set 13];5(2):19-24. Disponível em: http://bit.ly/2mZ8Nml

30. Godoy SCB. Absenteísmo-doença entre funcionários de um Hospital Universitário [dissertação]. Belo Horizonte: Universidade Federal de Minas Gerais; 2001.

31. Bezerra EL. Absenteísmo injustificado na enfermagem hospitalar [trabalho de conclusão de curso]. Londrina: Universidade Estadual de Londrina; 2008.

32. Gutierrez BAO, Ciampone MHT. O processo de morrer e a morte no enfoque dos profissionais de enfermagem de UTIs. Rev Esc Enferm USP [Internet]. 2007 [citado em 2016 ago 22];41(4):660-7. Disponível em: http://bit.ly/2nIlDZr

33. Sancinetti TR, Gaidzinski RR, Felli VEA, Fugulin FMT, Baptista PCP, Ciampone MHT, et al. Absenteísmo: doença na equipe de enfermagem: relação com a taxa de ocupação. Rev Esc Enferm USP [Internet]. 2009 [citado em 2016 set 11];43(2)1277-83. Disponível em: http://bit.ly/2nhKa6C

34. Pereira CA, Miranda LCS, Passos JP. O estresse ocupacional da equipe de enfermagem em setor fechado. Rev de Pesq.: cuidado é fundamental online [Internet]. 2009 [citado em 2016 set 11];1(2):196-202. Disponível em: http://bit. ly/2nhNdMd

35. Fugulin FMT, Gaidzinski RR, Kurcgant P. Ausências previstas e não previstas da equipe de enfermagem das unidades de internação do HU-USP. Rev Esc Enferm USP [Internet]. 2003 [citado em 2016 ago 9];37(4):109-17. Disponível em: http://bit.ly/2mVMqxj 
36. Parada EO, Alexandre NMC, Benatti MCC. Lesôes ocupacionais afetando a coluna vertebral em trabalhadores de enfermagem. Rev Latino-Am Enferm [Internet]. 2002 [citado em 2016 set 9];10(1):64-9. Disponível em: http://bit. ly/2nIFXtM

37. Costa JC, Lima RAG. Luto da equipe: revelaçóes dos profissionais de enfermagem sobre o cuidado à criança/adolescente no processo de morte e morrer. Rev Latino-Am Enferm [Internet]. 2005 [citado em: 2016 set 22];13(2):151-7. Disponível em: http://bit.ly/2nWh8bc

38. Batista, ACF. Os sentimentos da equipe de enfermagem diante da morte de crianças e adolescentes hospitalizados [trabalho de conclusão de curso]. Porto Alegre: Universidade Federal do Rio Grande do Sul; 2010.

39. Paro D, Paro J, Ferreira DLM. O enfermeiro e o cuidar em oncologia pediátrica. Arq Ciênc Saúde [Internet]. 2005 [citado em 2016 set 23];12(3):151-7. Disponível em: http:// bit.ly/2nsIxDN

40. Kessler AI, Krug SBF. Do prazer ao sofrimento no trabalho da enfermagem: o discurso dos trabalhadores. Rev Gaúcha Enferm [Internet]. 2012 [citado em 2016 out 25];33(1):4955. Disponível em: http://bit.ly/2nWliQo

41. Kovacs MJ. Sofrimento da equipe de saúde no contexto hospitalar: cuidando do cuidador profissional. O Mundo da Saúde [Internet]. 2010 [citado em 2016 set 3];34(4):420-9. Disponível em: http://bit.ly/2mZlJZm

42. Santos PG. O estresse e a síndrome de burnout em enfermeiros bombeiros atuantes em unidades de pronto-atendimento (UPA's) [dissertação] [Internet]. Rio de Janeiro: Universidade Federal do Estado do Rio de Janeiro; 2010 [citado em 2016 set 14].

43. Monteiro MS, Alexandre NMC, Rodrigues CM. Doenças músculo-esqueléticas, trabalho-esqueléticas, trabalho e estilo de vida entre trabalhadores de estilo de uma instituição pública de saúde. Rev Esc Enferm USP [Internet]. 2006 [citado em 2016 out 13];40(1):20-5. Disponível em: http:// bit.ly/2mVIBIh

44. Murofuse NT, Marziale MHP. Trastornos mentales y de comportamiento en trabajadores de enfermería de 23 instituciones de salud en Brasil. Rev Enferm IMSS [Internet]. 2005 [citado em 2016 out 12];13(3):133-40. Disponível em: http://bit.ly/2oRPI5O

45. Lelis CM, Battaus MRB, Freitas FCT, Rocha FLR, Marziale MHP, Robazzi MLCC. Distúrbios osteomusculares relacionados ao trabalho em profissionais de enfermagem: revisão integrativa da literatura. Acta Paul Enferm [Internet]. 2012 [citado em 2016 out 12];25(3):477-82. Disponível em: http://bit.ly/2nsH7ZW
46. Leite PC, Silva A, Merighi MAB. A mulher trabalhadora de enfermagem e os distúrbios osteomusculares relacionados ao trabalho. Rev Esc Enferm USP [Internet]. 2007 [citado em 2016 set 23];41(2):287-91. Disponível em: http://bit. ly/2oRyllQ

47. Gurgueira GP, Alexandre NMC, Filho HRC. Prevalência de sintomas músculo-esqueléticos em trabalhadoras de enfermagem. Rev Latino-Am Enferm [Internet]. 2003 [citado em 2016 out 13];11(5):608-13. Disponivel em: http://bit. ly/2oa10lx

48. Ceolin SUB, Marisco NS. Sofrimento psíquico em profissionais que atuam na rede básica de saúde, em um município do interior do Rio Grande do Sul [Internet]. XV Seminário Internacional de Educação no Mercosul. 2013 [citado em 2016 out 13]. Disponível em: http://bit.ly/2mzGBu8

49. Lima MCP. TMC e uso de álcool na população urbana de Botucatu - SP: um estudo de co-morbidade e utilização de serviços [tese]. São Paulo: Faculdade de Medicina da Universidade de São Paulo; 2004.

50. Fonseca MLG, Guimarães MBL, Vasconcelos EM. Sofrimento difuso e transtornos mentais comuns: uma revisão bibliográfica. Rev APS. 2008;11(3):285-94.

51. Seligmann-Silva E, Bernardo MH, Maeno M, Kato M. O mundo contemporâneo do trabalho e a saúde mental do trabalhador. Rev Bras Saúde Ocup [Internet]. 2010 [citado em 2016 out 12];35(122):187-91. Disponível em: http:// bit.ly/2nNF1EP

52. Chaves LD, Ramos LH, Figueiredo EN. Satisfação profissional de enfermeiros do trabalho no Brasil. Acta Paul Enferm [Internet]. 2011 [citado em 2016 ago 13];24(4):507-13. Disponível em: http://bit.ly/2nNqHwi

53. Tito RS. Burnout e transtornos mentais comuns nos trabalhadores de enfermagem que assistem crianças com cardiopatia grave [dissertação]. São Paulo: Escola de Enfermagem da Universidade de São Paulo; 2013.

54. Jacques MG. O nexo causal em saúde/doença mental no trabalho: uma demanda para a psicologia. Psicol Soc [Internet]. 2007 [citado em 2016 ago 13];19:112-9. Disponível em: http://bit.ly/2nNAsKO

55. Braga LC, Carvalho LR, Binder MCP. Condiçóes de trabalho e transtornos mentais comuns em trabalhadores da rede básica de saúde de Botucatu (SP). Ciênc Saúde Col [Internet]. 2010 [citado em 2016 set 21];15(1):1585-96. Disponível em: http://bit.ly/2mVMPj8

56. Alves M, Godoy SCB, Santana DM. Motivos de licenças médicas em um hospital de urgência-emergência. Rev Bras Enferm [Internet]. 2006 [citado em 2016 set 14];59(2):195200. Disponível em: http://bit.ly/2mxcVhe

\section{Como citar este artigo:}

Ayala ALM, Felicio ACR, Pachão J. Sofrimento dos profissionais que atuam no setor de oncologia em um hospital público de Joinville, SC. Rev. Aten. Saúde. 2017;15(51):106-117. 